\title{
EFICÁCIA DA ACUPUNTURA PARA O TRATAMENTO DA FIBROMIALGIA: uma revisão sistemática
}

DOI: $10.22289 / 2446-922 X . V 4 N 1 A 5$

\author{
Nayara Cristina Silveira ${ }^{1}$ \\ Raphael Cezar Carvalho Martins
}

\section{RESUMO}

A fisioterapia e a acupuntura têm um papel de destaque na melhora, no controle da dor, no aumento ou manutenção das habilidades funcionais de pacientes com fibromialgia. Partindo dessa premissa, o objetivo geral deste estudo é realizar uma revisão sistemática para corroborar a eficácia e distinguir os efeitos da acupuntura como forma de tratamento para o alívio da dor e melhora da qualidade de vida de pessoas com fibromialgia. Foi realizada uma revisão sistemática de literatura, os artigos utilizados no estudo encontram-se disponíveis em banco de dados inseridos nas bases de dados: Scielo, Google acadêmico, Banco de teses da USP, além de periódicos nacionais e internacionais. Os autores estudados concordam que a acupuntura é eficaz, no sentido de reduzir expressivamente a intensidade da dor e a quantidade de pontos doloridos em paciente portador de fibromialgia, de modo a melhorar a sua qualidade de vida devolvendo-Ihe capacidade funcional e vitalidade para vivenciar diferentes contextos que solicitam estabilidade física, emocional e mental que, comumente é comprometido pelos processos dolorosos.

Palavras-chave: Acupuntura; Fibromialgia; Mecanismo de ação.

\section{ABSTRACT}

Physiotherapy and acupuncture have a prominent role in improving, controlling pain, increasing or maintaining the functional abilities of patients with fibromyalgia. Based on this premise, the overall objective of this study is to perform a systematic review to corroborate the efficacy and distinguish the effects of acupuncture as a form of treatment for pain relief and improvement of the quality of life of people with fibromyalgia. A systematic review of the literature was carried out, the articles used in the study are available in databases included in the databases: Scielo, Google academic, Bank of theses of USP, as well as national and international journals. The authors agree that acupuncture is effective in reducing significantly the intensity of pain and the number of painful points in a patient with fibromyalgia, in order to improve their quality of life, giving them functional capacity and vitality to experience different contexts that call for physical, emotional and mental stability that is commonly compromised by painful processes.

Key words: Acupuncture; Fibromyalgia; Mechanism of action.

\footnotetext{
${ }^{1}$ Endereço eletrônico de contato: nayaraadvice@hotmail.com

Recebido em 16/10/2017. Aprovado pelo Conselho Editorial e aceito para publicação em 16/11/2017.
} 


\section{INTRODUÇÃO}

Não é fácil definir o que é acupuntura, isso devido a essa técnica elevar o Taoísmo, cuja filosofia considera a harmonia do indivíduo com o meio ambiente em que ele vive. Não se trata apenas de colocar agulha e curar enfermidades. Até mesmo porque no alicerce filosófico da acupuntura, o ideal é prevenir doenças. É oferecer uma diversidade de orientações para o paciente, que também, não é apenas o tratamento, o acupunturista deve trabalhar com orientações para o estilo de vida da pessoa. Assim, pode-se dizer que a acupuntura é parte da medicina chinesa (Massière, 2011).

A acupuntura, portanto, "é um recurso terapêutico do sistema médico conhecido no mundo ocidental como Medicina Tradicional Chinesa (MTC)." (Pereira, 2005, p.5). A MTC é realizada através de agulhas especiais, confeccionadas com ouro ou prata. As agulhas são colocadas em pontos especiais específicos do corpo, intitulados pontos de acupuntura, que são situados sobre linhas fictícias chamadas meridianos. Comumente, a acupuntura é recomendada para curar e aliviar diferentes tipos de dores (Silva, 2011).

A Organização Mundial da Saúde (OMS) reconhece o uso da acupuntura para diferentes tipos de enfermidades, como problemas psicossomáticos, enxaquecas, doenças gastrointestinais, alergias e múltiplas naturezas de dores, esta técnica, portanto, pode ser combinada com outras terapias (Vectore, 2005).

A fibromialgia, por exemplo, é uma síndrome crônica e dolorosa e, por essa razão, acaba por comprometer a qualidade de vida da pessoa, podendo dificultar as suas atividades cotidianas. No tratamento convencional da fibromialgia são utilizados, de forma crônica, medicamentos para controlar a dor. A Fisioterapia é uma possibilidade de tratamento para o controle da dor e sintomas valendo-se de exercícios leves, relaxamento e fortalecimento muscular para aliviar as dores e melhorar o sono. Mas, existem ainda as terapias alternativas que, em países mais desenvolvidos tem muita procura pela maior parte dos pacientes. A acupuntura vem sendo utilizada com muita frequência para o tratamento de dor, uma vez que, que pode contribuir para o equilíbrio adequado do corpo (Sanita, Moraes \& Santos, 2014).

Nessa perspectiva, busca-se compreender duas questões, primeira se a acupuntura pode ser uma forma eficaz de tratamento para o alívio da dor das pessoas com fibromialgia e segunda quais são os efeitos da acupuntura para as pessoas com fibromialgia. Partiu-se da suposição de que a acupuntura pode ser eficaz para reduzir a dor, melhorar o sono e a qualidade de vida dos portadores de fibromialgia.

Propõe-se este estudo na certeza de sua contribuição para o acervo da biblioteca da Faculdade Patos de Minas, que conta que poucos estudos nessa área. Para os estudantes 
de Fisioterapia e profissionais interessados em informações referentes ao tema proposto. Bem como, para os pacientes que têm fibromialgia e que desconhecem tratamentos específicos como a acupuntura, cujo método pode contribuir para melhorar as suas qualidades de vida.

O objetivo geral deste estudo é realizar uma revisão sistemática para corroborar a eficácia e distinguir os efeitos da acupuntura como forma de tratamento para o alívio da dor e melhora da qualidade de vida de pessoas com fibromialgia.

\section{MÉTODO}

Foi realizada uma revisão sistemática de literatura que "trata-se de um tipo de investigação focada em questão bem definida, que visa identificar, selecionar, avaliar e sintetizar as evidências relevantes disponíveis" (Galvão \& Pereira, 2014)

Os artigos utilizados no estudo encontram-se disponíveis em banco de dados inseridos nas bases de dados: Scielo, Google acadêmico, Banco de teses da USP, além de periódicos nacionais e internacionais.

Para o referencial teórico foram selecionadas 30 referências cujos objetivos fossem relacionados ao tema abordado e, por conseguinte, às seguintes palavras-chave: fibromialgia; acupuntura, efeitos. Os critérios de inclusão foram estudos relacionados para aos efeitos da acupuntura como forma de tratamento absoluto ou coadjuvante para as pessoas com fibromialgia publicados nos anos de 1985 a 2014. Foram selecionados estudos publicados em Português (31) e Inglês (1), ou seja, 32 estudos consultados.

Para a confecção da revisão sistemática a partir das evidências mais relevantes foram escolhidos para análise dos resultados 09 artigos que apresentaram como metodologia: estudo de caso, randomizado, prospectivo, e/ou observacional, publicados nos anos de 2007 a 2014.

\section{MARCO CONCEITUAL}

Acupuntura $(\mathrm{acu}=$ agulha + puntura $=$ picar $)$ procede da Medicina Tradicional Chinesa - MTC que se refere a uma técnica milenar, cujos registros somam mais de cinco mil anos. Trata-se de uma arte e ciência de inserir agulhas na pele dos indivíduos, com a finalidade de curar, de forma a restituir a saúde ou precaver enfermidades (Batello, 2007).

MTC se compõe da moxibustão, do qi gong e da fitoterapia que se associam à acupuntura, seu desígnio é prevenir e tratar enfermidades através do equilíbrio das energias Rev. Psicol Saúde e Debate. Fev., 2018:4(1)85-105. 
rotativas no corpo. A acupuntura abrange "a integração mente-corpo como um círculo de interação entre os sistemas internos e os aspectos emocionais, passível de ser concretizado através de três tesouros, ou seja, a Essência, o Qi e a Mente" (Vectore, 2005).

Amenizar os sintomas das enfermidades sem usar medicamentos é uma das caraterísticas da acupuntura, cujas agulhas podem aplicadas, na face, no abdômen, nas costas e na língua. Quando aplicado nas mãos o tratamento é designado Quiropuntura ou Quiro-acupuntura, nas mãos os estímulos podem ser feitos através de massageadores, agulhas especiais, imãs ou sementes (Lara, 2013)

Existem 365 pontos tradicionais de acupuntura espalhados pelo corpo. Essa variedade de pontos quando, ligados entre si, formam os meridianos correspondentes a algum órgão interno, estes são, os canais de conexão existentes no corpo humano (Aguiar, 2009)

Meridianos são linhas em que se concentram os pontos de acupuntura, as quais, após o diagnóstico introduzem-se as agulhas milimétricas. Genericamente, os pontos de acupuntura se localizam em partes de referência anatômica, que requer exatidão para serem localizadas. Estes pontos são lugares de maior resistência elétrica, por isso, podem ser detectados por aparelhos elétricos como é o caso do amperímetro (Batello, 2007).

São meridianos fundamentais: "o do estômago $(E)$; baço-pancrêas $(B P)$; pulmão $(P)$; intestino delgado (ID); intestino grosso (IG); rim (R); bexiga (B); fígado (F); vesícula biliar (VB); Coração (C); circulação e sexualidade (CS); triplo aquecedor" (TA) (Aguiar, 2009).

As técnicas mais populares da acupuntura são: Ventosaterapia, que busca descongestionar o fluxo de energia dos meridianos mediante aplicação de película de plástico na pele. Maxoterapia, que incide em aplicar um bastão aceso cheio de erva medicinal Artemísia em alguns pontos de acupuntura. Auricoloterapia, em que a terapia é realizada nas orelhas. E acupuntura Abdominal Chinesa, em que são aplicadas agulhas na região do abdômen (Lara, 2013)

Os mecanismos de ação da acupuntura abrangem três modelos: (1) Modelo bioelétrico demonstra que as áreas cutâneas em que estão os pontos de acupuntura podem propiciar maior condutividade elétrica, proporcionar maior objetividade aos pontos e meridianos da acupuntura e fornecer aplicações práticas como a Eletroacupuntura: essa terapia apresenta os princípios da acupuntura, mas são usados aparelhos elétricos que, ligados às agulhas ou eletrodos, transportam estímulos a alguns pontos do corpo com o objetivo de oferecer equilíbrio ao fluxo de energia. E a Qi Ryodoraku: linha de conexão (canal) eletropermeável que liga os pontos eletropermeáveis. Quando os nervos simpáticos distribuídos pela superfície do corpo ficam excitados, essa rede de linhas Ryodoraku fica evidente. Já foram descobertas 24 linhas Ryodoraku. (2) Modelo neuroquímico nasceu da 
mediação neuroquímica do efeito analgésico da acupuntura, apesar de evidenciar propriedades terapêuticas da acupuntura, ainda devem substanciar muitos dos efeitos obtidos pelos acupunturistas. (3) Sinal X, a dificuldade de se esclarecer as ações da acupuntura por meio da bioquímica ou da biofísica fez aparecer o modelo do sinal $\mathrm{X}$ fundamentado na teoria da informação e na teoria dos sistemas (Pereira, 2005).

\section{MECANISMO DE AÇÃO DA ACUPUNTURA}

A acupuntura apresenta diversos mecanismos de ação sendo o mais bem descrito o mecanismo analgésico por meio dos opióides endógenos. A manifestação mais expressiva da ação analgésica da acupuntura é o uso na analgesia cirúrgica. Além dos opióides, destacase a ação segmentar dos pontos de acupuntura, a justaposição parcial com os pontos-gatilho miofasciais e a diminuição do tônus simpático. Mas, mesmo assim, persistem controvérsias e ceticismo em relação à acupuntura, por vezes, em razão da carência de informação a respeito da técnica e do grande enredamento metodológico para a prática e interpretação de ensaios clínicos controlados. As mais variadas patologias podem ser tratadas com acupuntura, muito embora comumente, esta técnica seja mais procurada para o tratamento de dor musculo-esquelética dado à popularização do mecanismo de ação por meio dos opióides endógenos (Farias, 2000).

A acupuntura contribui para aumentar a resistência do hospedeiro. Quando existe agressão externa, determinados sistemas orgânicos são danificados. Ocorre que existe uma regulação interna para garantir resistência à enfermidade. Assim, a acupuntura tende a exasperar estes mecanismos para que, em menor tempo, o equilíbrio e a saúde se restabeleçam. Há possibilidade ainda de se estimular por meio da acupuntura o hipotálamo, a hipófise e outras glândulas que agem na recuperação da pessoa. Além disso, a acupuntura pode regular e normalizar as funções orgânicas. As diferentes funções no corpo do homem são interrelacionadas. Se existe algum distúrbio capaz de alterar esse interrelacionamento, acontece à manifestação de sintomas e a doença pode se estabelecer. Então, a acupuntura agencia o metabolismo. O metabolismo é essencial na sustentação da vida. Em algumas condições de enfermidade, existe alteração do metabolismo de múltiplos órgãos, com procedente abatimento e deficiência do organismo (Wen, 1985). 


\section{OS PONTOS DE DIAGNÓSTICO DA MEDICINA CHINESA}

Existem vínculos instituídos entre os órgãos e vísceras, entre os tecidos superficiais e sensoriais do corpo (olho, nariz, língua, ouvido), de maneira que qualquer distúrbio naqueles ocasionará sinais reflexos nestes. Essa ação acaba por criar, no organismo, um desequilíbrio que poderá levar à disfunção fisiológica. Deste modo, na diagnose e no tratamento de uma enfermidade, a medicina chinesa analisa o corpo em totalidade, com seus sinais e sintomas. No diagnóstico e classificação das enfermidades, consideram-se os fatores etiológicos, a magnitude da reação do organismo, o local em que se localizam as alterações dos sintomas, a alteração do pulso, a alteração na morfologia da língua entre outros (Wen, 1985).

No que concerne à diagnose e ao tratamento, existem diferentes conceitos e princípios na medicina chinesa que são similares aos da medicina moderna; outros, portanto, são bastante diferentes. Referentes à diagnose, existem quatro métodos:

1) Inspecionar: abrange a observação do todo ou de partes específicas do corpo do paciente. Cabe salientar que: "[...] o uso do toque é fundamental para fazer um diagnóstico na medicina chinesa. A arte do toque na Medicina Tradicional Chinesa é altamente sofisticada e inclui apalpação das áreas doloridas e pontos de diagnóstico e a tomada do pulso do paciente" (Schoenbart \& Shefi, 2013).

2) Ouvir as queixas e sentir os odores evidenciados pelo paciente: isso envolve a percepção dos seguintes aspectos: voz, ruído na respiração, tosse, soluço, arroto, odores da boca, salivação, expectoração, urina, fluxo menstrual e eventuais corrimentos, mau hálito, expectoração, suor, fezes e urina

3) Questionar os dados: na medicina chinesa, examina-se duração, local e tipo de sinais e sintomas.

4) Examinar o físico e a pulsologia: a força com que se palpa o pulso pode apresentar diferentes intensidades, ou seja: leve, forte ou intermediária. Cada uma delas revela certos pormenores a respeito do estado dos meridianos analisados, por meio das ondas de pulsação (WEN, 1985)

Embora o pulso possa ser sentido em diferentes lugares, o local principal é a artéria radial do pulso. Cada pulso tem três posições correspondentes a diferentes órgãos. "O pulso esquerdo corresponde ao yin do coração, fígado e rins. O pulso direito dá informações sobre o yang dos pulmões, baço e rins" (Schoenbart \& Shefi, 2013).

Já na classificação das síndromes, deve-se observar as síndromes de excesso que distinguem o começo da doença e seu meio, enquanto que as síndromes de deficiência preponderam nas doenças crônicas e em seu estágio final. As Síndromes de Deficiência se dividem em quatro categorias.

Rev. Psicol Saúde e Debate. Fev., 2018:4(1)85-105. 
a) Deficiência de Yin - Se existe ausência de Yin, existe demasia de Yang. Seus sinais e sintomas compreendem: calor, rubor facial, boca seca, mãos e pés quentes, aflição, sudorese, obstipação, urina escassa e amarelada. A língua fica avermelhada e por vezes apresenta fissuras. O pulso rápido, fino e fraco.

b) Deficiência de Yang - Sugere igualmente excesso de Yin; Além dos sinais de deficiência Yin, constata-se respiração fraca, pulso profundo e fino.

c) Deficiência de Qui (respiração ou energia) - Existe uma carência de energia, dispnéia aos esforços com taquipnéia exercional, tontura, fraqueza para falar, suor excessivo, maior quantidade de urina, prolapso anal ou do útero. A língua apresenta cor tênue com escassa camada superficial. O pulso fica fraco.

d) Deficiência de Hsue (sangue) - Evidenciam-se tontura e visão turva, agonia, insônia, sudorese noturna, obstipação, descoramento facial ou com cor amarelada, sem brilho. A boca se evidencia esbranquiçada. Em mulheres, pode acontecer amenorréia e fluxo menstrual escasso e de cor esmaecida (Wen, 1985).

Face ao exposto, ressalta-se que um histórico médico completo para realizar o diagnóstico do paciente é tão relevante para a Medicina Tradicional Chinesa como para a medicina ocidental. Pois, podem ser evidenciadas pistas esclarecedoras dos problemas presentes. Torna-se importante também observar o uso de quaisquer medicamentos sob prescrição, visto que os sintomas do paciente podem ser em virtude dos efeitos colaterais desses medicamentos (Schoenbart \& Shefi, 2013).

\section{CONDIÇÃO DA ACUPUNTURA NO BRASIL}

Desde que foi inserida na área da saúde no Brasil, a acupuntura vem passando por diferentes fases. Nas décadas de 1960 e 1970 distingue-se a fase pré-institucional da acupuntura no país, cujo interesse da área da saúde pela acupuntura é muito reduzido, sem uma organização eficaz. A década de 1980 foi um marco na organização institucional, profissional $\mathrm{e} o$ ingresso da acupuntura no ambiente público de assistência à saúde. A década de 1990 procedeu na decorrência do extenso reconhecimento da acupuntura como especialidade médica (Rocha, Tolentino, Genschow \& Sampaio, 2008).

Em meados de 1995, o Conselho Federal de Medicina (CFM) reconhece a acupuntura como especialidade médica exclusiva e passa a impedir a prática por outros profissionais. Mas, em cascata, a acupuntura foi sendo reconhecida como especialidade pelos Conselhos de Enfermagem, Fonoaudiologia, Psicologia, Farmácia, Educação Física, Odontologia e Medicina Veterinária. Enfatiza-se que o Conselho de Fisioterapia e Terapia 
Ocupacional foi pioneiro ao reconhecer a acupuntura como especialidade de seus membros, seguido pelo Conselho de Biomedicina (Massière, 2011).

Muitas foram às tentativas de monopólio dessa atividade por parte do CFM, mas, no Brasil, compete à União legislar o exercício de profissões. Na justiça, houve muitas denúncias contra acupunturistas que por não serem formados em medicina são taxados de "leigos" sem considerar desconsiderando vastas experiências e aprendizado da MTC. Apesar disso, não tem alcançado êxito e o Superior Tribunal de Justiça (STJ) designou jurisprudência adepta à liberdade da prática, como não há lei que regulamenta o exercício da acupuntura, o STJ avalia que ninguém tem obrigação de deixar de exercê-la (Massière, 2011)

Também, conforme a Constituição Federal Brasileira (BRASIL, 1988) "é livre o exercício de qualquer trabalho, ofício ou profissão, atendidas as qualificações profissionais que a lei estabelecer".

\section{FIBROMIALGIA}

A fibromialgia é uma doença reumatológica que se manifesta através da dor musculoesquelética difusa e crônica. No Brasil, é a segunda doença reumatológica mais frequente, após a osteoartrite. A doença acomete cerca de $2,5 \%$ da população, sendo mais comum sua manifestação em mulheres, principalmente com idades entre 35 e 44 anos. Embora reconhecida há muito tempo, a fibromialgia passou a ser mais estudada nas últimas três décadas e até o momento, não se conhecem tratamentos que sejam considerados muito eficazes (Heymann, Paiva, Milton, Pollak, Martinez et al. 2010)

Caracterizada por dores musculoesqueléticas difusas, a fibromialgia desencadeia vários pontos musculares dolorosos, que são detectados pelo exame físico. A definição da síndrome ocorreu no final da década de 1970 com a descrição de manifestações clínicas e dos sintomas gerais e psíquicos. Os critérios se mostraram deficientes ao longo dos anos, pois a palpação correta dos pontos representa um desafio, principalmente na atenção primária. Acredita-se que seja necessária nova abordagem para a síndrome, seja através da anamnese, do exame físico, ou do raciocínio diagnóstico (Oliveira, Petean \& Louzana Júnior, 2010)

Tida como uma das causas mais comuns de dor musculoesquelética crônica, a fibromialgia tem o diagnóstico predominantemente clínico, uma vez que não há exames que comprovem o quadro. O tratamento da fibromialgia é diverso e várias ações são simultaneamente recomentadas, tais como atividade física aeróbica, tratamento das 
alterações do sono, analgesia efetiva, bem como o tratamento das condições associadas, como a depressão (Paiva, 2007)

As causas da fibromialgia, de fato, são desconhecidas, no entanto existem vários fatores associados à síndrome, tais como: genética; infecções por vírus e doenças autoimunes; distúrbios do sono; sedentarismo; ansiedade e depressão. Os principais sintomas associados à fibromialgia são: dor generalizada; fadiga; dificuldades cognitivas; dor de cabeça recorrente ou enxaqueca clássica, dor pélvica e dor abdominal sem causa identificada; problemas de memória e de concentração; dormência e formigamento nas mãos e nos pés; palpitações; e ainda redução na capacidade de se exercitar (Sociedade Brasileira para Estudos da Dor, 2017).

Para o diagnóstico devem-se considerar todos os sinais e sintomas, considerando ainda as afecções secundárias. Desse modo, o seu diagnóstico deve ser fundado na observação clínica com auxílio de exames subsidiários (Martinez, 2016). A fibromialgia, a depender do quadro possui diferentes classificações, conforme mostra a Tabela 1.

Por não ser totalmente entendida a terapêutica da fibromiolgia permanece empírica, havendo diversas formas de tratamento, sem que nenhuma consiga resultados considerados excelentes. As principais drogas utilizadas no tratamento são amitriptilina, a imipramina, a clomipramina e a doxepina, devido a capacidade que possuem de inibir a reutilização da serotonina. Porém, para um tratamento efetivo, o uso da medicação deve ser combinado com uma mudança de hábitos de vida e pela prática regular de esportes, requerendo a participação do paciente, assumindo uma postura ativa em relação à doença (Marques, Matsutani \& Ferreira, 2002).

É importante conhecer alternativas para o tratamento efetivo da fibromialgia que possam diminuir o impacto da doença sobre a qualidade de vida dos pacientes devido às limitações funcionais dela decorrentes. Os impactos dos sintomas, por afetar diversas áreas são tão importantes quanto à doença em si, razão pela qual o tratamento atual está voltado, principalmente, para a redução dos sintomas (Marques, Matsutani \& Ferreira, 2002).

Cita-se, como exemplo, o estudo de Martins (2008), cujo objetivo foi comparar a associação da acupuntura ao alongamento muscular passivo com o alongamento muscular passivo isolado, no ganho de amplitude de movimento, flexibilidade e na melhora do quadro álgico na região da coluna lombar em alunos do ensino superior com a faixa etária entre 20 e 30 anos devidamente matriculados na FPM - Faculdade Patos de Minas em Patos de Minas - MG. O trabalho foi realizado com uma amostra de 15 alunos divididos em três grupos randomizados compostos por cinco alunos em cada grupo. No grupo $A$, foram realizadas cinco sessões de tratamento por acupuntura; no grupo B, foram realizadas cinco sessões de 
tratamento por cinesioacupuntura; e finalmente no grupo $C$, foram realizadas cinco sessões de tratamento por cinesioterapia, em síntese o autor ressalta que:

A associação da acupuntura com técnicas da cinesioterapia é uma proposta inovadora quando o objetivo da abordagem terapêutica é a realização de um diagnóstico e tratamento de patologias musculoesqueléticas, como a dor na coluna lombar, mesclando a visão holística da acupuntura com a visão reducionista de técnicas ocidentais tradicionais. (...) Pode-se concluir com este estudo que a associação da acupuntura ao alongamento muscular passivo (cinesioacupuntura) quando comparada ao alongamento muscular passivo e à acupuntura isolada se mostrou estatisticamente relevante na melhora da flexibilidade e na redução do quadro álgico na região da coluna lombar; apesar do alongamento muscular passivo ter se apresentado como sendo a melhor técnica a ser usada com este objetivo (Martins, 2008)

Classificações Características

1. Fibromialgia primária

2. Fibromialgia secundária

3. Fibromialgia regional ou localizada

4. Fibromialgia do idoso

5. Fibromialgia infanto juvenil
Achados característicos de fibromialgia sem uma causa subjacente reconhecível.

Achados característicos secundários a uma causa conhecida ou a uma doença subjacente e que apresenta melhora dos sintomas fibromiálgicos com o tratamento específico da condição de base.

Dor miofascial localizada associada com a presença de trigger points, geralmente secundária a distensões musculares (ocupacional, repetitiva); bastante similar as síndromes miofasciais específicas locais ou regionais, não preenche os critérios definidos para fibromialgia, porém considerada por alguns autores como uma forma frustra.

Similar à fibromialgia primária. Atenção especial para o diagnóstico diferencial com polimialgia reumática, doenças neurológicas degenerativas, osteoporose, síndromes paraneoplásicas, doença de Parkinson inicial, síndromes cerebrais orgânicas e síndromes pós virais que cursam com fadiga pronunciada.

Similar às formas primárias, ocorrendo em crianças e adolescentes.

Tabela 1 - Classificações da fibromialgia 
Nesse contexto, a Fisioterapia e a acupuntura têm um papel de destaque na melhora, no controle da dor e no aumento ou manutenção das habilidades funcionais do paciente, bem como na redução de outros sintomas que causam sofrimento (Marques, Matsutani \& Ferreira, 2002).

\section{RESULTADOS E DISCUSSÃO}

$\mathrm{Na}$ Tabela 1 apresenta-se a revisão sistemática realizada. Foram selecionados artigos cujas características fossem relacionadas aos efeitos da acupuntura como forma de tratamento absoluto ou coadjuvante para as pessoas com fibromialgia.

\begin{tabular}{|c|c|c|c|c|}
\hline Autor/Ano & Objetivo & Metodologia & Resultados & Conclusão \\
\hline $\begin{array}{l}\text { Stival et al., } \\
\text { (2014). }\end{array}$ & $\begin{array}{l}\text { Avaliar a eficácia } \\
\text { da acupuntura no } \\
\text { tratamento da } \\
\text { fibromialgia, } \\
\text { considerando-se } \\
\text { como desfecho } \\
\text { primário a } \\
\text { resposta } \\
\text { imediata da } \\
\text { escala visual } \\
\text { analógica (VAS) } \\
\text { para avaliação } \\
\text { da dor. }\end{array}$ & $\begin{array}{l}\text { Estudo } \\
\text { randomizado, } \\
\text { controlado e duplo- } \\
\text { cego incluindo } 36 \\
\text { pacientes } \\
\text { portadores de } \\
\text { fibromialgia (ACR } \\
\text { 1990) selecionados } \\
\text { no ambulatório de } \\
\text { Reumatologia da } \\
\text { Santa Casa de } \\
\text { Misericórdia de } \\
\text { Ponta Grossa, PR. }\end{array}$ & $\begin{array}{l}\text { A variação entre o valor da } \\
\text { EVA final e da EVA inicial } \\
\text { foi de }-4,36 \pm 3,23 \text { ( } P= \\
0,0001) \text { no grupo de } \\
\text { tratamento e de }-1,70 \pm \\
1,55 \text { no grupo de controle } \\
(P=0,06) \text {. A diferença na } \\
\text { amplitude de variação da } \\
\text { EVA (EVA inicial }- \text { final) } \\
\text { entre os grupos favoreceu } \\
\text { o procedimento } \\
\text { verdadeiro ( } P=0,005) \text {. O } \\
\text { tamanho de efeito (effect } \\
\text { size-ES) para o grupo de } \\
\text { tratamento foi de d }=1,7,0 \\
\text { que é considerado um } \\
\text { efeito grande. Embora } \\
\text { com uma amostra } \\
\text { reduzida, seu poder } \\
\text { estatístico para esses } \\
\text { resultados foi bastante } \\
\text { relevante (94,8\%). }\end{array}$ & $\begin{array}{l}\text { A acupuntura mostrou } \\
\text { ser eficaz na reduc,ão } \\
\text { imediata da dor em } \\
\text { pacientes portadores } \\
\text { de fibromialgia, com } \\
\text { um tamanho de efeito } \\
\text { (effect size) bastante } \\
\text { significativo }\end{array}$ \\
\hline Araújo (2007) & $\begin{array}{l}\text { O objetivo desse } \\
\text { estudo foi avaliar } \\
\text { o benefício da } \\
\text { acupuntura } \\
\text { associada ao } \\
\text { tratamento } \\
\text { convencional } \\
\text { com }\end{array}$ & $\begin{array}{l}\text { Estudo prospectivo } \\
\text { com } 58 \text { pacientes } \\
\text { do sexo feminino } \\
\text { com idades } \\
\text { entre } 27 \text { e } 70 \text { anos } \\
\text { (média } 51,70 \pm \\
\begin{array}{l}10,97), \quad \text { que } \\
\text { apresentavam dor } \\
\text { de }\end{array}\end{array}$ & $\begin{array}{l}\text { No final das vinte sessões, } \\
\text { as pacientes que } \\
\text { receberam a acupuntura } \\
\text { apresentaram melhora } \\
\text { significante nas medidas } \\
\text { de dor (EVA, NPD e IM) e } \\
\text { em cinco sub-escalas do } \\
\text { SF36 em relação ao grupo } \\
\text { controle. Após seis meses } \\
\text { o grupo de acupuntura } \\
\text { apresentou resultado }\end{array}$ & $\begin{array}{lr}\text { A associação } & \text { da } \\
\text { acupuntura } & \text { ao } \\
\text { tratamento } & \\
\text { convencional } & \text { da } \\
\text { fibromialgia } & \text { com } \\
\text { antidepressivos } & \\
\text { tricíclicos e exercícios } \\
\text { é benéfica r para } \\
\text { doentes r com } \\
\text { fibromialgia, ro } \\
\text { período de três } & \text { meses }\end{array}$ \\
\hline
\end{tabular}

Rev. Psicol Saúde e Debate. Fev., 2018:4(1)85-105. 


\begin{tabular}{|c|c|c|c|c|}
\hline & $\begin{array}{l}\text { antidepressivos } \\
\text { tricíclicos e } \\
\\
\text { exercícios } \quad \text { em } \\
\text { pacientes com } \\
\text { fibromialgia }\end{array}$ & 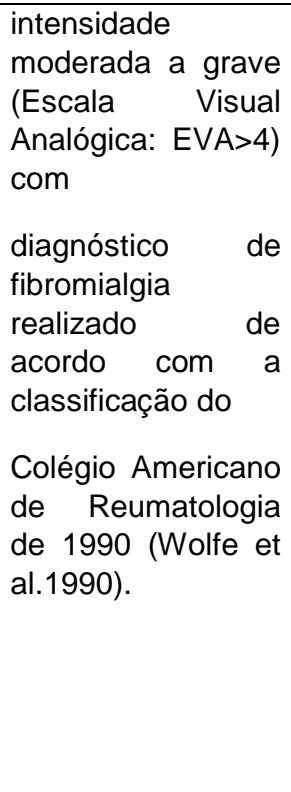 & $\begin{array}{l}\text { melhor do que o controle } \\
\text { em relação ao NPD e ao } \\
\text { IM e em uma sub-escala } \\
\text { do SF36. Após um ano o } \\
\text { grupo de acupuntura } \\
\text { mostrou melhora sobre o } \\
\text { grupo controle somente } \\
\text { em uma sub-escala do } \\
\text { SF36. Depois de dois } \\
\text { anos do início do } \\
\text { tratamento com } \\
\text { acupuntura não houve } \\
\text { diferença significativa } \\
\text { entre os dois grupos em } \\
\text { todas as medidas } \\
\text { pesquisadas. }\end{array}$ & $\begin{array}{l}\text { após o término do } \\
\text { tratamento. } \\
\text { tratamento } \\
\text { complementar de } \\
\text { acupuntura reduz a } \\
\text { intensidade da dor, o } \\
\text { número de pontos } \\
\text { dolorosos e o índice } \\
\text { miálgico em doentes } \\
\text { com fibromialgia. O } \\
\text { uso da acupuntura } \\
\text { melhora a qualidade } \\
\text { de vida de doentes } \\
\text { com fibromialgia nos } \\
\text { seguintes domínios: } \\
\text { capacidade funcional, } \\
\text { dor, vitalidade, } \\
\text { aspectos social e } \\
\text { emocional e a saúde } \\
\text { mental. }\end{array}$ \\
\hline Autor/Ano & Objetivo & Metodologia & Resultados & Conclusão \\
\hline $\begin{array}{l}\text { Takiguchi, et } \\
\text { al. (2008) }\end{array}$ & $\begin{array}{l}\text { Verificar } \\
\text { eficácia da } \\
\text { acupuntura na } \\
\text { melhora da dor, } \\
\text { sono e qualidade } \\
\text { de vida de } \\
\text { pacientes } \\
\text { fibromiálgicas. }\end{array}$ & 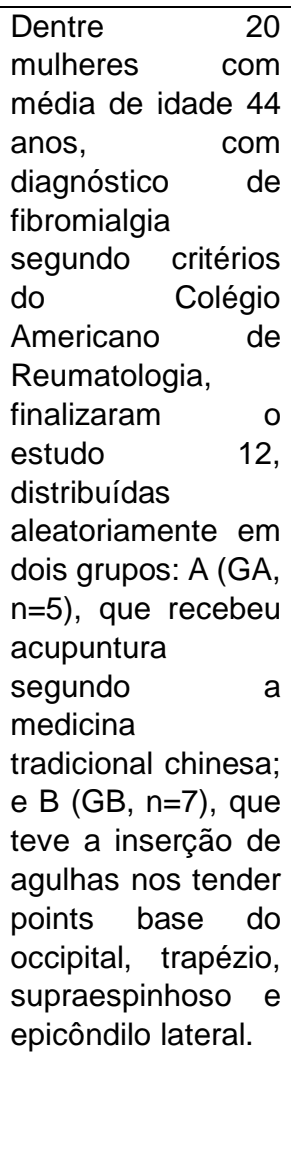 & $\begin{array}{l}\text { A dor foi avaliada por } \\
\text { escala visual analógica e } \\
\text { dolorimetria; o sono, pelo } \\
\text { Inventário do Sono; e a } \\
\text { qualidade de vida, pelo } \\
\text { Questionário de Impacto } \\
\text { da Fibromialgia (QIF). Os } \\
\text { grupos receberam } \\
\text { acupuntura uma vez por } \\
\text { semana, durante oito } \\
\text { semanas, com inserção } \\
\text { de oito agulhas por } 25 \\
\text { minutos. Os dados foram } \\
\text { tratados estatisticamente, } \\
\text { adotandose o nível de } \\
\text { significância a=0,05. No } \\
\text { GA, houve melhora } \\
\text { estatisticamente } \\
\text { significante do sono; e no } \\
\text { GB, na dor, limiar de dor } \\
\text { nos tender points, no sono } \\
\text { e nos itens do QIF dor, } \\
\text { cansaço matinal, } \\
\text { ansiedade e depressão }\end{array}$ & $\begin{array}{l}\text { A diminuição do limiar } \\
\text { de dor no grupo B, } \\
\text { mesmo em pontos } \\
\text { onde não foi feita a } \\
\text { acupuntura, } \\
\text { demonstra o efeito } \\
\text { sistêmico da inserção } \\
\text { de agulhas, causando } \\
\text { alívio da dor mesmo } \\
\text { em locais distantes. A } \\
\text { qualidade do sono } \\
\text { parecer estar } \\
\text { intimamente ligada a } \\
\text { sintomas como a dor } \\
\text { crônica, sendo difícil } \\
\text { determinar qual } \\
\text { desses dois sintomas } \\
\text { seria a causa e qual a } \\
\text { conseqüência. Como } \\
\text { no grupo B não foram } \\
\text { puncionados pontos } \\
\text { específicos envolvidos } \\
\text { com o sono, infere-se } \\
\text { que or efeito } \\
\text { analgésico obtido, } \\
\text { aliado à liberação de } \\
\text { substâncias } \\
\text { "semelhantes ao ópio“ } \\
\text { no fluido cérebro- } \\
\text { espinhal. }\end{array}$ \\
\hline $\begin{array}{l}\text { Roriz, et al., } \\
(2008)\end{array}$ & $\begin{array}{l}\text { Verificar a } \\
\text { eficácia da TENS } \\
\text { de } \quad \text { baixa } \\
\text { frequência e alta } \\
\text { intensidade na }\end{array}$ & $\begin{array}{l}\text { Estudo de caso da } \\
\text { paciente R. S. S., } \\
38 \text { anos de idade, } \\
\text { sexor feminino } \\
\text { portadora da }\end{array}$ & $\begin{array}{l}\text { Houve uma redução } \\
\text { significativa da } \\
\text { intensidade da dor em } \\
77,7 \% \text { dos tender points } \\
\text { na segunda avaliação e de }\end{array}$ & $\begin{array}{l}\text { Conclui-se que houve } \\
\text { uma redução da dor } \\
\text { dos tender points da } \\
\text { paciente, } \\
\text { evidenciando a }\end{array}$ \\
\hline
\end{tabular}

Rev. Psicol Saúde e Debate. Fev., 2018:4(1)85-105. 


\begin{tabular}{|c|c|c|c|c|}
\hline & $\begin{array}{l}\text { modulação dor } \\
\text { dos tender points } \\
\text { dos pacientes } \\
\text { com fibromialgia. }\end{array}$ & $\begin{array}{l}\text { síndrome de } \\
\text { fibromialgia } \\
\text { atendida na clínica } \\
\text { escola } \\
\text { Faculdade } \\
\text { Integrada do } \\
\text { (FISIOFIC). } \\
\text { paciente } \\
\text { submetida } \\
\text { tratamento com a } \\
\text { Estimulação } \\
\text { Elétrica Nervosa } \\
\text { Transcutânea } \\
\text { (TENS) } \\
\text { acupuntura nos } \\
\text { pontos dolorosos } \\
\text { num total de doze } \\
\text { atendimentos e a } \\
\text { dor avaliada antes } \\
\text { de iniciar } \\
\text { tratamento e após } \\
\text { cada tra } \\
\text { atendimentos. }\end{array}$ & $\begin{array}{l}88,8 \% \text { dos pontos nas } \\
\text { demais avaliações. }\end{array}$ & $\begin{array}{l}\text { analgesia promovida } \\
\text { pela TENS, portanto } \\
\text { ela deve ser utilizada } \\
\text { como uma terapia } \\
\text { complementar } \\
\text { associada a outros } \\
\text { programas } \\
\text { tratamentos e serviu } \\
\text { também como uma } \\
\text { boa técnica para } \\
\text { localizar os tender } \\
\text { points. }\end{array}$ \\
\hline Autor/Ano & Objetivo & Metodologia & Resultados & Conclusão \\
\hline $\begin{array}{l}\text { Oliveira; } \\
\text { Sousa e } \\
\text { Godoy (2014) }\end{array}$ & $\begin{array}{l}\text { O objetivo do } \\
\text { estudo foi avaliar } \\
\text { o efeito da } \\
\text { acupuntura na } \\
\text { atividade } \\
\text { eletrodermal, na } \\
\text { qualidade de vida } \\
\text { e na redução da } \\
\text { dor em indivíduos } \\
\text { fibromiálgicos. }\end{array}$ & 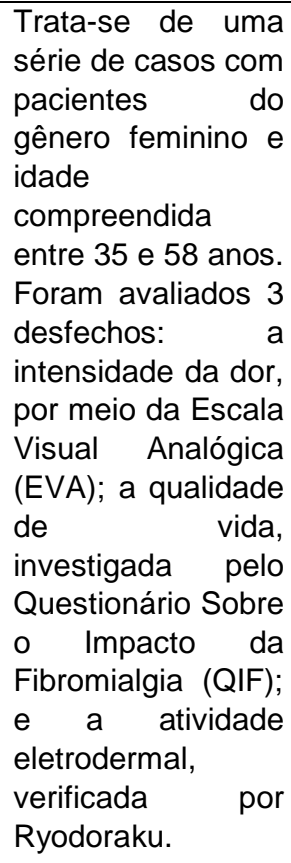 & $\begin{array}{l}\text { Como } \\
\text { observou-se resultado, } \\
\text { percentual da dor entre } \\
34 \% \text { a } 63 \% \text { na EVA, } \\
\text { melhora em } 7 \text { dos } 10 \text { itens } \\
\text { do QIF e aumento da } \\
\text { eletropermeabilidade } \\
\text { média do Ryodoraku, de } \\
34,95 \pm 11,12 \quad \text { para } \\
46 \pm 11,66 .\end{array}$ & $\begin{array}{l}\text { Conclui-se que a } \\
\text { acupuntura promoveu } \\
\text { melhoria dos sintomas } \\
\text { em todas as } \\
\text { participantes do } \\
\text { estudo. O desfecho } \\
\text { primário do estudo foi } \\
\text { que o tratamento com } \\
\text { a acupuntura reduziu } \\
\text { a intensidade da dor } \\
\text { em todas as } \\
\text { participantes. } \\
\text { Secundariamente, } \\
\text { notou-se que houve } \\
\text { melhora em sete dos } \\
\text { dez itens do } \\
\text { Questionário Sobre o } \\
\text { Impacto da } \\
\text { Fibromialgia e que a } \\
\text { atividade eletrodermal } \\
\text { aproximou-se da domeostase. }\end{array}$ \\
\hline
\end{tabular}




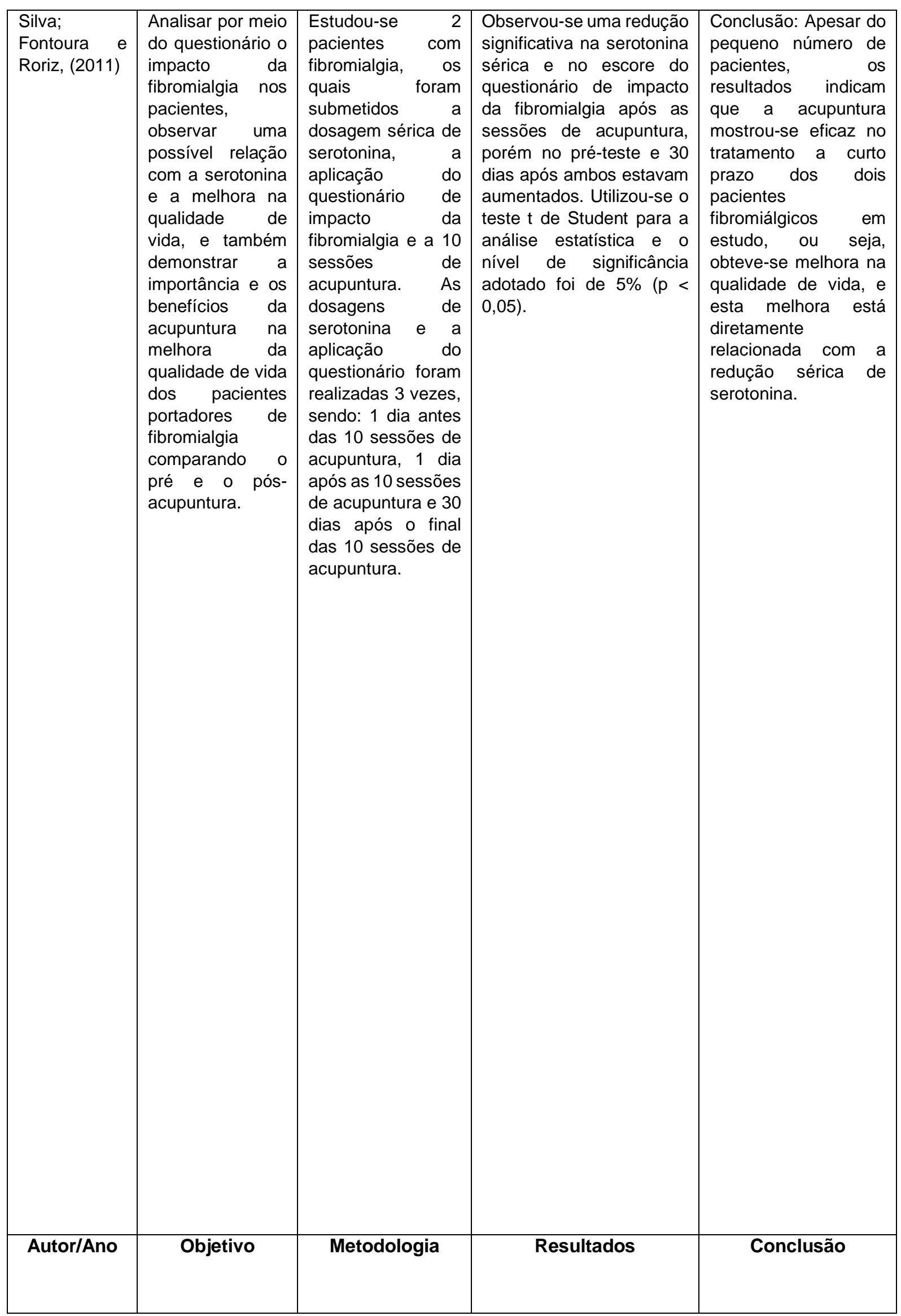

Rev. Psicol Saúde e Debate. Fev., 2018:4(1)85-105. 


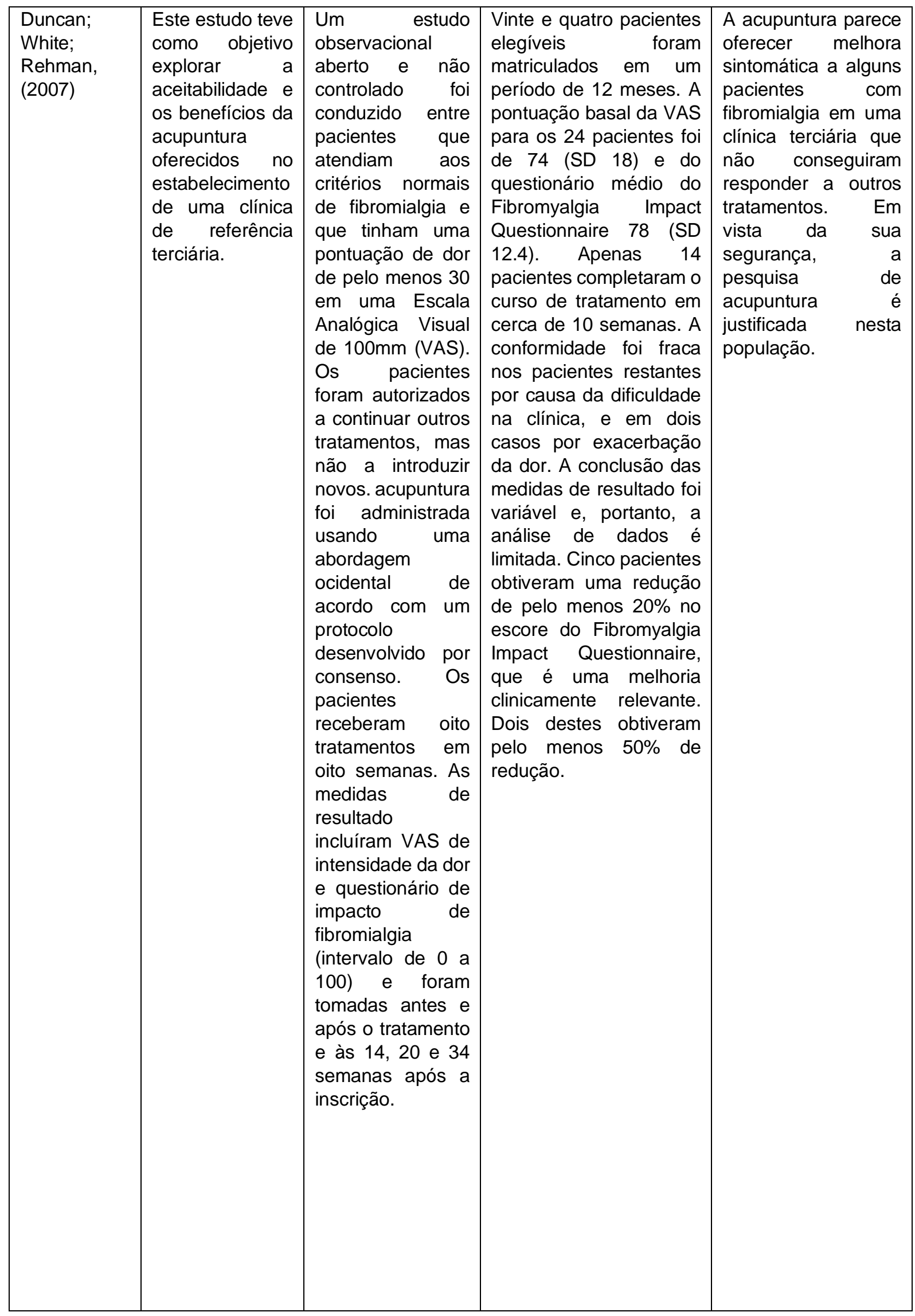




\begin{tabular}{|c|c|c|c|c|}
\hline Autor/Ano & Objetivo & Metodologia & Resultados & Conclusão \\
\hline $\begin{array}{l}\text { Bastos, et al., } \\
(2013)\end{array}$ & $\begin{array}{l}\text { Este estudo teve } \\
\text { como objetivo } \\
\text { investigar se a } \\
\text { acupuntura em } \\
\text { pontos sensíveis } \\
\text { poderia } \\
\text { efetivamente } \\
\text { gerir o FMS. }\end{array}$ & 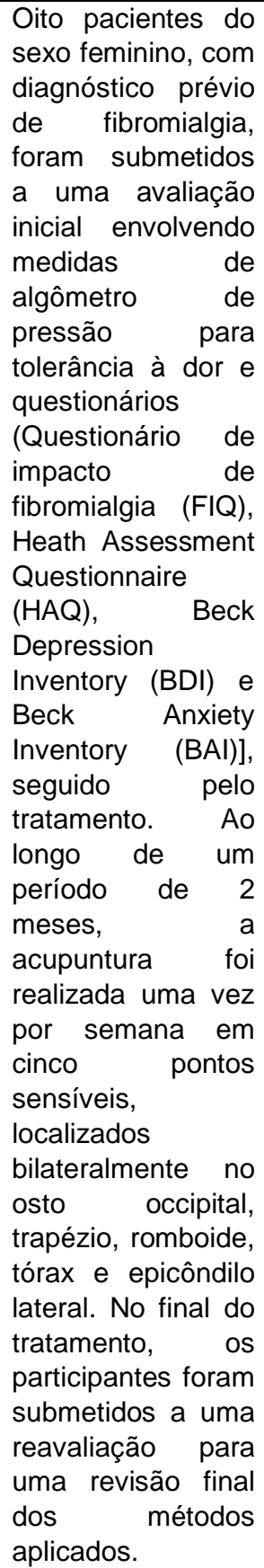 & $\begin{array}{l}\text { Observamos uma redução } \\
\text { no limiar de dor e } \\
\text { sensibilidade e melhora } \\
\text { nas áreas de ansiedade e } \\
\text { depressão e qualidade de } \\
\text { vida, que foram } \\
\text { demonstradas usando } \\
\text { FIQ, BDI e BAI, mas não o } \\
\text { HAQ. }\end{array}$ & $\begin{array}{l}\text { Os resultados } \\
\text { demonstraram a } \\
\text { eficácia do tratamento } \\
\text { de acupuntura do } \\
\text { ponto tênue no bem- } \\
\text { estar geral dos } \\
\text { pacientes, não só pela } \\
\text { melhoria da qualidade } \\
\text { de vida, mas também } \\
\text { pela redução da } \\
\text { sensibilidade à dor do } \\
\text { FMS }\end{array}$ \\
\hline
\end{tabular}

Rev. Psicol Saúde e Debate. Fev., 2018:4(1)85-105. 


\begin{tabular}{|c|c|c|c|c|}
\hline Autor/Ano & Objetivo & Metodologia & Resultados & Conclusão \\
\hline $\begin{array}{l}\text { Targino, et al. } \\
(2008)\end{array}$ & $\begin{array}{l}\text { Avaliar a eficácia } \\
\text { da acupuntura } \\
\text { para fibromialgia. }\end{array}$ & $\begin{array}{l}\text { Cinquenta e oito } \\
\text { mulheres com } \\
\text { fibromialgia foram } \\
\text { alocadas } \\
\text { aleatoriamente } \\
\text { para receber } \\
\text { acupuntura em } \\
\text { conjunto com } \\
\text { antidepressivos } \\
\text { tricíclicos es } \\
\text { exercício ( } n=34 \text { ), } \\
\text { ou antidepressivos } \\
\text { tricíclicos e apenas } \\
\text { exercícios ( } n=24) \text {. } \\
\text { Os pacientes } \\
\text { avaliaram sua dor } \\
\text { em uma escala } \\
\text { analógica visual. } \\
\text { Um avaliador cego } \\
\text { avaliou o valor do } \\
\text { limiar de dor de } \\
\text { pressão média em } \\
\text { todos os } 18 \text { pontos } \\
\text { de fibromialgia e } \\
\text { qualidade de vida }\end{array}$ & $\begin{array}{l}\text { No final de } 20 \text { sessões, os } \\
\text { pacientes que receberam } \\
\text { acupuntura foram } \\
\text { significativamente } \\
\text { melhores do que o grupo } \\
\text { controle em todas as } \\
\text { medidas da dor em } 5 \text { das } \\
\text { subescalas SF-36. Após } 6 \\
\text { meses, O grupo de } \\
\text { acupuntura foi } \\
\text { significativamente melhor } \\
\text { do que o grupo controle } \\
\text { em números de pontos } \\
\text { macios, limiar médio de } \\
\text { dor de pressão nos } 18 \\
\text { pontos de concurso e } 3 \\
\text { subescalas de SF-36. } \\
\text { Após um ano, o grupo de } \\
\text { acupuntura mostrou } \\
\text { significância em uma } \\
\text { subescala do SF-36; Aos } \\
2 \text { anos, não houve } \\
\text { diferenças significativas } \\
\text { nas medidas de resultado. }\end{array}$ & $\begin{array}{lr}\text { A adição } & \text { de } \\
\text { acupuntura } & \text { aos } \\
\text { tratamentos } & \text { usuais } \\
\text { para fibromialgia pode } \\
\text { ser benéfica para dor } \\
\text { e qualidade de vida } \\
\text { por } 3 \text { meses } & \text { após o } \\
\text { término do tratamento. } \\
\text { Pesquisas futuras são } \\
\text { necessárias } & \text { para } \\
\text { avaliar os } & \text { efeitos } \\
\text { específicos } & \text { da } \\
\text { acupuntura } & \text { para a } \\
\text { fibromialgia. } & \text { Aos } 2 \\
\text { anos, não } & \text { houve } \\
\text { diferenças } & \\
\text { significativas } & \text { nas } \\
\text { medidas de resultado. }\end{array}$ \\
\hline
\end{tabular}

Tabela 2 - Revisão sistemática

A análise da Tabela 2 permite pontuar as seguintes considerações:

Silva; Fontoura \& Roriz (2011) concordam que os resultados indicam que a acupuntura pode ser eficaz no tratamento em curto prazo de pacientes fibromiálgicos, e vão além, pois, explicam que a melhora na qualidade de vida tem relação direta com a redução sérica de serotonina.

Bastos et al. (2013) acrescentam que os resultados do seu estudo demonstraram a eficácia do tratamento de acupuntura para o bem-estar geral dos pacientes, não só pela melhoria da qualidade de vida, mas também pela redução da sensibilidade à dor.

Takiguchi et al. (2008) observou que houve redução de dor mesmo em pontos onde não foi realizada a acupuntura, fato que evidencia o efeito sistêmico da inserção de agulhas, causando alívio da dor mesmo em locais distantes.

Araújo (2007) sugere associar acupuntura, ao tratamento convencional da fibromialgia com antidepressivos tricíclicos e atividade física.

Roriz et al. (2008) também sugerem associação de tratamentos para aliviar os sintomas da fibromialgia, os autores sugerem, por exemplo, associar a acupuntura e o TENS (Estimulação Nervosa Elétrica Transcutânea) mas, esta última deve ser utilizada como uma terapia complementar. 
Duncan, White \& Rehman (2007) em correspondência, comentam que a acupuntura sozinha, parece oferecer melhora sintomática a alguns pacientes com fibromialgia, que não conseguiram responder a outros tratamentos.

Targino et al. (2008) concordam que a adição de acupuntura aos tratamentos usuais para fibromialgia pode ser benéfica para dor e qualidade de vida por 3 meses após o término do tratamento.

Stival et al. (2014) complementa afirmando que a acupuntura, aplicada aos padrões da Medicina Tradicional Chinesa, possui efeito importante na redução imediata da dor em pacientes com fibromialgia quando comparada aos efeitos da acupuntura simulada.

Duncan, White \& Rehman (2007), Takiguchi et al. (2008), Araújo (2007), Silva, Fontoura \& Roriz (2011), Bastos et al. (2013), Oliveira, Sousa \& Godoy (2014), Stival et al. (2014) concordam que a acupuntura é eficaz para reduzir a intensidade da dor, a quantidade de pontos doloridos e o índice miálgico em pacientes portadores de fibromialgia, de forma a melhorar a sua qualidade de vida.

Targino et al. (2008) alertam que pesquisas futuras são necessárias para avaliar os efeitos específicos da acupuntura para a fibromialgia, pois aos 2 anos, não houve diferenças significativas nas medidas de resultado.

Stival et al. (2014) também concordam que mais estudos com diferentes protocolos são imprescindíveis, com maior número de indivíduos e com maior tempo de sequência, especialmente com o objetivo de avaliar sobremodo mais preciso a duração do efeito analgésico da acupuntura.

\section{CONSIDERAÇÕES FINAIS}

O presente estudo apresentou uma revisão sistemática para corroborar a eficácia e distinguir os efeitos da acupuntura como forma de tratamento para o alívio da dor e melhora da qualidade de vida de pessoas com fibromialgia. A hipótese levantada para o estudo foi confirmada, pois, os autores estudados concordam que a acupuntura é eficaz, no sentido de reduzir significativamente a intensidade da dor e a quantidade de pontos doloridos em paciente portador de fibromialgia, de forma a melhorar a sua qualidade de vida devolvendoIhe capacidade funcional e vitalidade para vivenciar diferentes contextos que requerem estabilidade física, emocional e mental que, normalmente é afetado pelos processos dolorosos. Mas, esses achados não descartam a necessidade de se realizar estudos maiores e mais complexificados para ser possível obter maiores esclarecimentos sobre o tema. 


\section{REFERÊNCIAS}

Aguiar, D. F. (2009). Abordagem terapêutica da acupuntura em portadoras de cefaleia do tipo tensional crônica. Belém. Trabalho de Conclusão de Curso (Graduação) Universidade da Amazônia. Centro de Ciências Biológicas e da Saúde. Curso de Fisioterapia, Amazônia, Brasil.

Antônio, S. F. (2000). Fibromialgia. Rev. Brasil. Medicina, 58(12):1-7.

Araújo, R. A. T. (2007). Tratamento da dor na fibromialgia com acupuntura. São Paulo, Tese (Doutorado) - Faculdade de Medicina, Universidade de São Paulo, São Paulo, Brasil.

Battello, C. F. acupuntura, o que é? para pacientes e interessados. Santo André, SP: Editora Cartex, 2007.

Brasil. (1988) Constituição da República Federativa do Brasil: promulgada em 5 de outubro de 1988. Contém as emendas constitucionais posteriores. Brasília, DF: Senado.

Duncan, B. White, A. Rahman (2007). A. Acupuncture in the treatment of fibromyalgia in tertiary care - a case series. Acupuncture In Medicine, 25(4):137-147.

Bastos, J. L., Pires, E. D., Silva, M. L., Araújo, F. L., Silva, J. R. (2013). Effect of Acupuncture at Tender Points for the Management of Fibromyalgia Syndrome: A Case Series. J Acupunct Meridian Stud, 6(3):163-8.

Farias, F. P. (2000). Abordagem científica da acupuntura no tratamento de doenças geriátricas. Disponível em: <http://www.bva.med.br/bva/acervo/apresentacoes/geriatriaforum2001.ppt.>. Acesso em: 13 set. 2017.

Galvão, T. F., Pereira, M. G. (2014). Revisões sistemáticas da literatura: passos para sua elaboração. Epidemiol. Serv. Saúde. 23(1):183-184.

Heymann, R. E., Paiva, E. S. P., Milton, H. J., Pollak, D. F., Martinez, J. E., et al. (2010). Consenso brasileiro do tratamento da fibromialgia. Rev. Bras. Reumatol. 50(1):56-66.

Lara, S. acupuntura auxilia no tratamento de doenças. Terapia oriental investiga não apenas o problema, mas também a origem de todos eles Medicina Alternativa. Ed. 412.2013 .2 Disponível em: http://www.correiodeltapetiningacom.br/Portal/correiodeitapetininga2013012535095> . Acesso em: 13 set. 2017. 
Martinez, J. E. (2006). Fibromialgia: O Desafio do Diagnóstico Correto. Rev. Bras. Reumatol. 46(1):1-2.

Marques, A. P., Matsutani, L. A., Ferreira, E. A. G, M. L. L. F. (2002). A fisioterapia no tratamento de pacientes com fibromialgia: uma revisão de literatura. Rev. Bras. Reumatol. 42(1):1-7.

Martins, R. C. C. (2008) Promovendo saúde através da acupuntura associada ao alongamento passivo na redução da dor lombar e no ganho de flexibilidade em estudantes universitários. Mestrado (Fisioterapia e Terapia Ocupacional). Programas de Pós-graduação da CAPES. Unifran. Promoção de saúde, Franca, São Paulo, Brasil.

Massière, L. A. (2011). Significação da acupuntura no Brasil: percalços de uma ecologia de saberes no ensino de saúde e seus reflexos na profissionalização. Dissertação (Mestrado). Universidade Federal de Pernambuco, CFCH. Programa de Pós-Graduação em Sociologia. Recife.

Oliveira, R. M., Sousa, H. Á., Godoy J. R. P. (2014). Efeito da acupuntura na qualidade de vida e no tratamento da dor em pacientes com fibromialgia. Universitas: Ciências da Saúde. 12(1):37-48.

Oliveira, R. D. R., Petean, F. C., Louzana Júnior, P. (2010). Fibromialgia. RBM. Como Diagnosticar e Tratar. Moreira Júnior. 67(1):1-5.

Paiva, E. S. Fibromialgia. (2007). Revis. Brasil. Medicina. Moreira Júnior, 64(12):1-9.

Pereira, F. A. O. (2005). Evidências científicas da ação da acupuntura. Perspectivas, Campos dos Goytacazes. 4(7):88-105.

Rocha, D. K., Tolentino, B. G., Genschow, F. C. Z; Sampaio, F. C. (2008). acupuntura Médica no Brasil - Um Breve Histórico. Colégio Médico Brasileiro de acupuntura.

Roriz, I. M. M., Farias, M. S. Q., Barros, G. G., Câmara, T. M. S., Sousa, C. T. , Bastos, V.P. D. (2008) Estimulação elétrica nervosa transcutânea na modulação da dor dos tender points na síndrome de fibromialgia: estudo caso. Rev. Saúde. Com., 4(2):177189.

Sanita, P. C. V. M., Moraes, T. S. M, Santos, F. M. (2014). Análise do efeito da acupuntura no tratamento da fibromialgia: revisão de literatura. Manual Therapy, Posturology e Rehabilitation Journal. MTP e Rehab Journal. (12):188-204.

Silva, M. E. F, Mejeia, D. P. M. (2011). acupuntura e doença psicossomática. Disponível em: <http://www.portalbiocursos.com.br/artigos/acupuntura/39.pdf>. Acesso em: 13 set. 2017. 
Schoenbart, B., Shefi, E. (2013). Uso do toque no diagnóstico da Medicina Tradicional Chinesa. Disponível em: < http://saude.hsw.uol.com.br/medicina-chinesadiagnose5.htm />. Acesso em: 13 set. 2017.

Sociedade Brasileira para Estudos da Dor (SBED). Fibromialgia. (2017). Disponível em: < http://www.sbed.org.br/lermais_materias.php?cd_matérias=473>. Acesso em: 05. Jun. 2017.

Silva, F., Fontoura, H. S, Roriz, C. F. V. (2011). A utilização da acupuntura no tratamento da fibromialgia relacionando com a qualidade de vida e a dosagem sérica de serotonina. Fisioterapia Brasil, 12(4):298-302.

Scotton, A. S., Fraga, R. O. (2000) Como diagnosticar e tratar fibromialgia. Rev. Brasil. Medicina. 57(12):1-6.

Stival, R. S. M., Cavalheiro, P. R., Stasiak, C. E. S, Galdino, D. T., Hoekstraa, B. E. H, Schafransk, M. D. (2004). acupuntura na fibromialgia: um estudo randomizadocontrolado abordando a resposta imediata da dor. Rev. Bras. Reumatol, 54(6):431-36.

Takiguchi, R. S, Fukuhara, V. S, Sauer, J. F., Assumpção, A., Marques, A. P. (2008). Efeito da acupuntura na melhora da dor, sono e qualidade de vida em pacientes fibromiálgicos: estudo preliminar. Fisioterapia e Pesquisa. 15(3):280-4.

Targino, R. A., Imamura, M., Kaziyama, H. H., Souza, L. P., Hsing, W. T., et al. Azevedo Neto, R. S. (2008). A Randomized Controlled Trial of Acupuncture Added to Usual Treatment for Fibromyalgia. Journal of Rehabilitation. Medicine, 40(7):582-588.

Vectore, C. (2005) Psicologia e acupuntura: Primeiras Aproximações. Psicologia Ciência e Profissão. 25(2):266-285.

Wen, T. S. (1985). acupuntura Clássica Chinesa. São Paulo: Editora Cultrix. 